\section{Polio in Maharashtra}

A 11 month old child has been confirmed to have vaccine derived polio virus (VDPV) in the Beed district of Maharashtra on June $1^{\text {st }} 2013$. This is the third case of VDPV since March 2012 and the first in Maharashtra. There is a suspicion of underlying immunodeficiency in the child since he has been suffering from prolonged bouts of illnesses. India will not forfeit the polio free label because of this since VDPV is not enumerated in wild polio numbers. Stool samples of people in the neighborhood have been sent to National Institute of Virology, Pune to evaluate extent of spread. The next plan is 2-3 rounds of immunization rounds which is recommended to break the extension of disease even in VPVD (The Times of India 7 June 2013).

\section{Reconstructing the Bronchus with a 3-D Printer}

Life threatening episodes of cyanosis due to severe bronchomalacia in an infant has been treated with an artificially created bronchus and reported in the 22 May issue of the New England Journal of Medicine. Scientists used an incredible new technology called 3-D printing. So far severe bronchomalacia has been treated with intermittent ventilation, artificial intraluminal splints and sometimes even surgical splinting with a rib. However all of these have their limitations and drawbacks. Glen Green, an otorhinolaryngologist from the University of Michigan, had been working for some time to develop a custom made tube to support floppy airways.

A 3-D printer works like an inkjet printer, but instead of laying down layers of ink it deposits a structural material. The printer head adds each layer according to a digital pattern to create a 3-D structure. In manufacturing, 3-D printers have built prototypes and parts for machines. So far artificial ears, and spinal disks and bones have been created at an experimental level in the laboratory. But this is the first time a 3-D printer has been used to aid tissue reconstruction in a patient. The 3-D printer heats up a powdered form of the plastic until it melts and can be extruded in a paste which is made up of a reabsorbable material called polycaprolactone.

The team first used a computed tomography (CT) scan to define the patient's airways. From those images, they then sculpted a three-dimensional printed cast with the same shape as the patient's collapsed bronchus. Hollister, a professor of biomedical engineering at Michigan then designed a sleeve that would wrap around the outside of the floppy airway. The sleeve's structure allows it to expand as the airway grows and develops while simultaneously resisting spasms that pull inward, thereby collapsing the airway. The next step was to sew the tissue of the patient's bronchus to the inside of the sleeve. The team needed to obtain an emergency-use approval from the U.S. Food and Drug Administration before they could implant the device. The surgery was done in January 2012 and the child has been asymptomatic since then. The next big challenge will be printing fully functioning organs and tissues for humans (Nature 28 May 2013).

\section{Marvelous Mucus}

Mucus mainly consists of huge molecular complexes called mucins, which are made up of thousands of glycan sugars attached to a central protein backbone. This mucus is loaded heavily with bacteria killing viruses called phages. While phages protect their hosts from the constant barrage of bacteria, these very bacteria are an ideal breeding ground for the phages to reproduce. Researchers have shown that the presence of phages reduced the number of bacteria that can attach to mucus by more than 10,000 times. It is suspected that phage strains found most often in mucus will be those that target the most common bacteria, providing a sort of 'mucus memory' against the most relevant local microbes. Mucusborne phages also possibly protect bacteria that benefit their animal hosts, while destroying those that cause harm. The clinical implications are that deliberately coating a tissue with phage loaded mucus would boost the basal immunity and may open a whole new chapter in therapeutics (Nature 20 May 2013).

\section{Healing The City}

Nearly $40 \%$ of Indians will be living in urban centers by 2026 . A quarter of them will be poor and $10 \%$ vulnerable (ie rag pickers, homeless children etc) with negligible access to health care services. So, the Union Cabinet's approval of Rs 22, 507 crore for the National Urban Health Mission was long overdue. Other highlight of the NUHM is the inclusion of other urban centric issues such as domestic violence, sexual exploitation, HIV/AIDS, drug trafficking, child abuse and gender issues. While the Centre will fund 75 per cent of the mission and the State 25 per cent, the funding ratio for North Eastern States and special category States of Jammu and Kashmir, Himachal Pradesh and Uttarakhand will be 90:10. The existing mechanism and systems created and functioning under NRHM will be strengthened to meet the needs of NUHM (The Hindu 21 May 2013).

Gouri Rao Passi gouripassi@hotmail.com 